Relations industrielles

Industrial Relations

\title{
Les cadres du secteur privé en Belgique, par Maurice Piraux, Collection « Etudes sociales ", nos 41-42. La Pensée catholique, Bruxelles, Office général du livre, Paris, 1961. 104 pages.
}

\section{Gérard Dion}

Volume 16, numéro 4, octobre 1961

URI : https://id.erudit.org/iderudit/1021693ar

DOI : https://doi.org/10.7202/1021693ar

Aller au sommaire du numéro

Éditeur(s)

Département des relations industrielles de l’Université Laval

ISSN

0034-379X (imprimé)

1703-8138 (numérique)

Découvrir la revue

Citer ce compte rendu

Dion, G. (1961). Compte rendu de [Les cadres du secteur privé en Belgique, par Maurice Piraux, Collection « Etudes sociales ", nos 41-42. La Pensée catholique, Bruxelles, Office général du livre, Paris, 1961. 104 pages.] Relations industrielles / Industrial Relations, 16(4), 509-510. https://doi.org/10.7202/1021693ar

Tous droits réservés (C Département des relations industrielles de l’Université Laval, 1961
Ce document est protégé par la loi sur le droit d'auteur. L'utilisation des services d'Érudit (y compris la reproduction) est assujettie à sa politique d'utilisation que vous pouvez consulter en ligne.

https://apropos.erudit.org/fr/usagers/politique-dutilisation/ 


\section{Management in the Industrial World,} by Frederick Harbison \& Charles $\mathbf{A}$. Myers. New York: McGraw-Hill Book Company, Inc., 1959. 413 pp. \$7.

Dans nos sociétés industrielles, la direction des entreprises a plusieurs dimensions (sociale, économique et politique) et est perçue de diverses façons (comme série de fonctions, comme classe d'élite, et ainsi de suite).

Le livre que voici, de Harbison et Myers, s'efforce de décrire le cadre logique du développement de la direction dans le contexte du processus de croissance industrielle. Il s'agit donc avant tout d'une étude dynamique, qui envisage la direction des entreprises d'abord comme une ressource économique, puis comme un système d'autorité, et enfin comme une classe ou élite.

Les auteurs mettent à profit l'insertion de leur étude dans le vaste projet international de l'\& Inter-University Study of Labor Problems in Economic Development », qui a étudié le phénomène de l'industrialisation dans une quarantaine de pays. Ils tâchent de présenter un concept de la direction des entreprises qui soit pertinent à toutes les phases du processus d'industrialisation.

Le première partie de l'ouvrage s'attache justement à la formulation d'un concept vraiment international de la direction. Le premier chapitre de cette partie situe la direction en regard de la théorie de l'organisation, et décrit les fonctions et la hiérarchie de la direction de même que le rôle du bâtisseur d'entreprise. Les trois autres chapitres de cette première partie examinent la direction comme une ressource, comme un cadre autoritaire et comme une classe.

La seconde partie, très considérable, est constituée de monographies parallèles qui rendent compte de l'organisation de la direction des entreprises dans onze pays du monde qui représentent adéquatement toutes les phases de l'industrialisation.

Il est très utile à tous, cadres, chefs syndicaux, hauts fonctionnaires et professeurs, de frotter leurs notions de la direction des entreprises avec la réalité internationale dont les deux auteurs rendent compte dans ce livre.

Roger Chartier
Les cadres du secteur privé en Belgique, par Maurice Piraux, Collection \&-Etudes sociales », nos 41-42. La Pensée catholique, Bruxelles, Office général du livre, Paris, 1961. 104 pages.

Même s'il en est qui continuent encore à utiliser les catégories marxistes dans le domaine des relations du travail, on sait que les termes\& patrons 》 et <ouvriers » n'ont pas beaucoup de sens. «Direction》, «personnel de cadre 》, «personnel de maîtrise », «employés 》, 《 ouvriers 》 sont des expressions que l'on entend de plus en plus. Elles correspondent à des réalités différentes que l'on se doit de définir de la façon la plus précise possible.

Cette étude sociologique ne porte que sur les «cadres». L'auteur nous montre d'abord que ce n'est pas une tâche facile que de préciser les limites de cette notion. Il s'arrête, cependant, en l'expliquant, à la définition suggérée par le Groupement National des Cadres:

« Est cadre le membre de l'entreprise qui, d'une part, par son statut iuridique est lié à celle-ci par contrat d'emploi et, d'autre part, par sa fonction, participe d l'activité de direction. Cette fonction est principalement caractérisée par 1) la conception et la mise en oeuvre des moyens destinés à réaliser la finalité de l'entreprise; 2) l'étendue de lautonomie, de la responsabilité qui lui sont conférées et la mesure dans laquelle par ses actes quelqu'un engage l'entreprise envers les tiers; 3) une formation intellectuelle ou technique équivalente à celle que donnent les études du degré supérieur. >

A partir d'un certain nombre d'enquêtes effectuées par différents organismes, l'auteur fait une étude-synthèse du phénomène «cadre 》 dans le secteur privé en Belgique. Il est ainsi amené à considérer les caractéristiques sociologiques des cadres; origine, âge, situation familiale, formation, études, embauche, fonction dans l'entreprise, durée de travail, mobilité, rémunération, mode de vie, loisir, engagement social, politique et syndical, etc.

Bien que la valeur proprement scientifique de cette étude soit infirmée parce qu'elle repose sur des enquêtes faites soit à des époques différentes ou avec des méthodes plus ou moins rigoureuses, elle n'en est pas moins très intéressante. Elle apporte des indications précieuses 
sur l'état des cadres dans le secteur privé de Belgique et provoquera, sans doute, des recherches plus poussées et plus méthodiques.

La littérature française en sociologie occupationnelle est tellement rare que nous ne pouvons pas ne pas saluer avec joie cette tentative d'analyser une catégorie dont l'importance est sans cesse grandissante.

\section{Gérard Dion}

Au service de la petite industrie, publication du Bureau International du Travail, Genève, 1961, 246 pp.

Rares sont les volumes s'attaquant aux problèmes de la petite industrie, quoique celle-ci «fournit de l'emploi à une fraction considérable de la maind'oeuvre industrielle et représente une forte proportion de l'ensemble des établissements industriels ».

Comme on l'indique dans l'introduction du volume, l'ouvrage est destiné avant tout aux personnes qui, du fait des responsabilités ou des fonctions qui sont les leurs, ou pour tout autre motif, se préoccupent de dispenser des conseils, de donner un enseignement ou de fournir d'autres services aux exploitants de petites entreprises industrielles. Il est aussi destiné, il va sans dire, aux chefs de petites entreprises eux-mêmes.

Après avoir défini la petite industrie et étudié son rôle dans le développement économique, on traite ensuite des caractéristiques et des besoins de la petite industrie, comme le financement, l'approvisionnement en matières premières, l'écoulement des produits, etc...

La partie la plus importante du volume étudie les « services de promotion technique » qui ont pour but de communiquer certaines connaissances et certaines informations aux directeurs et aux travailleurs de petits établissements. Leurs activités les plus importantes sont les consultations techniques, la formation professionnelle, l'information et la recherche.

Ces derniers chapitres portent sur l'aide financière et matérielle, et sur la contribution que peut apporter la coopération à la solution des problèmes de la petite industrie.
Cet ouvrage, comme toutes les autres publications du B.I.T. d'ailleurs, est excellent, et son grand mérite est de s'attaquer à des problèmes majeurs de la petite industrie qui sont trop négligés hélas, des experts du monde de l'industrie.

Jean-Paul Deschênes

«La femme au travail». Ouvrage en collaboration. Numéro spécial de la revue Esprit, 29ème année, no 295, mai 1961.

Le travail des femmes est une donnée majeure de notre époque, un fait acquis. On peut le regretter, cela ne changera rien. Il importe de l'analyser, d'en comprendre toutes les dimensions. C'est un fait récent dans l'histoire et complexe dans ses significations. Pour certains, c'est une promotion, alors que pour d'autres, c'est une forme d'esclavage. Le travail féminin est aussi un fait humain qui métamorphose la cellule familiale, modifie la distribution traditionnelle du travail entre hommes et femmes.

Ce numéro spécial d'Esprit qui tente d'évaluer le fait du travail féminin d'une manière objective a été réalisé grâce à la collaboration d'une dizaine de spécialistes en la matière. On y envisage les dimensions du problème, ses données biologiques, les contradictions sociales qu'il comporte et l'ambiguité de l'attitude feminine à son égard.

Pareille étude est indispensable à tous ceux qui veulent voir clair dans le problème du travail féminin.

GÉrard Dion

Industrialism and Industrial Man, by Clark Kerr, John T. Dunlop, Frederick Harbison and Charles A. Myers. Cambridge: Harvard University Press, 1960. 331 pp. $\$ 6$.

Voici en quelque sorte un volumesynthèse des travaux publiés à date sous l'étiquette de l'« Inter-University Study of Labor Problems in Economic Development ». Ce groupe financé largement par la Dotation Ford, se forma en 1954 autour des quatre économistes du travail Kerr (de Californie), Dunlop (de Harvard), Harbison (de Chicago, puis Princeton) et Myers (du M.I.T.), pour lesquels l'anthropologie, la sociologie et la psychologie sont des amies. 